\title{
IMPORTANCIA DE LA GESTIÓN REGIONAL DEL SANEAMIENTO: EL PARADIGMA DEL ESTADO DE SANTA CATARINA EN EL USO DEL SUBSIDIO CRUZADO
}

\author{
IMPORTANCE OF REGIONAL SANITATION MANAGEMENT: THE \\ PARADIGM OF THE STATE OF SANTA CATARINA IN THE USE OF \\ THE CROSS-SUBSIDY
}

(iD) Haneron Victor Marcos ${ }^{1 *}$, (iD) Bessy Castillo Santa María ${ }^{1}$

haneronmarcos@gmail.com; bsscstll@gmail.com

${ }^{1}$ Universidad César Vallejo, Lima, Perú

*Correspondencia: Haneron Victor Marcos. Email: haneronmarcos@gmail.com

Recibido: 10.09.2020 | Aprobado: 20.10.2020

\section{RESUMEN}

Este artículo pretende demonstrar la importancia del subsidio cruzado para la gestión de los servicios de saneamiento y la implementación de políticas públicas relacionadas, permitido por la administración regional. Tratase de un modelo mayoritariamente aplicado en Brasil - que se encuentra amenazado por privatizaciones y municipalizaciones de sistemas superavitarios, además por la falta de una percepción legislativa para una revisión de la titularidad, que en Brasil es deferida a los municipios en regla general (la excepción de regiones metropolitanas y aglomerados urbanos sigue sin reglamentación). Investigación metodológica de enfoque cualitativo, paradigma socio crítico y estudio de caso, que pasa por el escenario internacional con soportes de autores como Bel (2019), BorjaVega y Kloeve (2018), Helm (2019), y sustentada por paradigmas sudamericanos presentados por Bonifaz Fernández y Montoya (2013), Casa (2013), y Ceppi (2014), además de los nacionales como Paludo y Borba (2014), Borja (2014), Cruz y Ramos (2016), Souza y Costa (2016) y Brown et al. (2016). En conclusión, la quiebra del modelo solidario de subsidio entre ciudades ya es una realidad brasileña, especialmente en el Estado de Santa Catarina (Brasil), y que los ejemplos locales de privatización no han evidenciado éxito diferencial, exigiendo un reposicionamiento estratégico del mismo para que la universalidad del acceso a los servicios de saneamiento, con modicidad tarifaria, sea una realidad tangible.

Palabras clave: Gestión del saneamiento, privatización, subsidio cruzado, Santa Catarina.

\begin{abstract}
This article aims to demonstrate the importance of cross-subsidy for the management of sanitation services and the implementation of related public policies, allowed by the regional administration. This is a model - mostly applied in Brazil - that is threatened by the privatization and municipalization of surplus systems, as well as by the lack of a legislative perception for a revision of legal competence, which in Brazil is deferred to municipalities in general rule (the exception of metropolitan areas and urban agglomerations continues without regulation). This is a methodological investigation with a qualitative approach, a socio-critical paradigm and a case study, which passes through the international stage with the support of authors such as Bel (2019), Borja-Vega and Kloeve (2018), Helm (2019), and supported by South American paradigms presented by Bonifaz Fernández and Montoya (2013), Casa (2013), and Ceppi (2014), in addition to nationals such as Paludo and Borba (2014), Borja (2014), Cruz and Ramos (2016), Souza and Costa (2016) and Brown et al., (2016). In conclusion, the bankruptcy of the solidarity model of subsidy between cities is already a Brazilian reality, especially in the State of Santa Catarina (Brazil), and that the local examples of privatization have not evidenced differential success, demanding a strategic repositioning of it so that the universality of access to sanitation services, with tariff modicity, is a tangible reality.
\end{abstract}

Keywords: Sanitation management; privatization; cross-subsidy. 


\section{INTRODUCCIÓN}

En nuestra sociedad, guiada, dentro del "contrato social", por principios legales, éticos y morales, existe una miscelánea de normas positivadas. No todas son (o debían ser) necesarias, lo que se refleja en el servicio de saneamiento. Es evidente su esencialidad y su afectación a la dignidad humana; constituyendo un verdadero fin de derechos humanos, que debe alcanzar a todos, independiente de clases sociales, u otra clasificación construida de manera autoritaria o artificial por la sociedad. Cuando se habla de saneamiento, hay referencia a los servicios de abastecimiento de agua potable de coleta y depuración de aguas residuales o alcantarillado.

Por eso, surge en la mente cuándo recordamos la iniciativa boliviana en la Asamblea General de la Organización de las Naciones Unidas (ONU, 2010) explicada por la cosmovisión de los pueblos indígenas sobre los recursos naturales y por la sensibilidad que revestía la problemática para el pueblo boliviano tras el episodio de la guerra del agua en Cochabamba en el año 2000 (Ceppi, 2014). No era necesario - o no debería ser, por la obviedad - que este país "tercermundista", merecedor del apoyo internacional, fuese a Nueva York clamar por el reconocimiento de esos derechos en cuanto derechos humanos.

Sin embargo, nuestro modelo positivista, de subjetividades y omisiones para la superación del más fuerte sobre lo más débil, exige convicción y previsión expresa para una mayor seguridad (tanto que hubo 41 abstenciones, de países importantes como EUA, Suecia, Australia, Japón, incluso el Reino Unido, mucho en función de la incompatibilidad del reconocimiento con la agenda y principios neoliberales (Brown et al., 2016)). Además, las verdades necesitan ser repetidas, y así hubo la aprobación del reclamo a través de la Resolución No. 64/292, que en un mundo con casi un billón de personas sin acceso al agua potable y más que el doble sin acceso al saneamiento básico (Borja-Vega, 2018), intenta ser un nuevo faro al fomento de inversiones internacionales.

Aunque es evidente el esfuerzo internacional, la normativa no ha sido suficiente para la garantía de acceso a ese derecho; en Brasil, tal como en la gran mayoría de los países de la América del Sur, el modelo vigente en Brasil es el del subsidio cruzado (por productos, clases de consumidores y ciudades), con servicios dirigidos por compañías estatales responsables de la administración regional, aunque la titularidad (o la competencia legal) sea, ordinariamente, municipal por disposición constitucional.

El subsidio cruzado, entre ciudades, es el objeto de estudio, y responde como herramienta para a corrección de distorsiones regionales, en que los sistemas superavitarios socorren a los deficitarios, creando un modelo solidario, cuya importancia se presenta aún más revelada en un ambiente de pandemia, que se hace exigir una mayor presencia y eficacia de los servicios de saneamiento. En consecuencia, está enmarcada a resultados donde la municipalización y privatización están imponiendo una revisión del modelo y una necesidad de adaptación. Es necesario responder cuál es el impacto de su fin en la corrección de las distorsiones regionales en materia de saneamiento y salud pública.

El objetivo es determinar el subsidio cruzado en la gestión de los servicios de saneamiento y la implementación de políticas públicas relacionadas a la administración 
regional y específicamente los riesgos al modelo por creciente fenómeno de la privatización y de la municipalización de los sistemas.

\section{EL ESCENARIO HISTÓRICO}

Para transponer el panorama macro al micro, de resolución a la cuestión planteada, es necesario conocer el escenario de la gestión del sector en Brasil, y los fenómenos que lo afectan y que son relacionados con el objetivo propuesto.

Sin embargo, es importante conocer el aporte histórico mundial (Orta 2018). Desde el inicio de estos servicios hasta mediado del siglo XIX, el predominó de un modelo privatista, preocupado con el soporte a los sectores de mayor poder adquisitivo. Un segundo momento, comienza a finales del siglo XIX y principios del siglo XX, señalado por procesos de estatización, delante la incapacidad de respuesta del modelo a la ascendiente urbanización. En ese momento, aplicase la "lógica racional administrativa" (Orta, 2018, p. 154) en que los servicios toman la lógica de los derechos sociales garantizados por el Estado, independiente de la condición financiera de los usuarios, siendo este un servicio público en esencia.

En la década del 1990, especialmente en América Latina, las reformas neoliberales impulsadas por el Consenso de Washington asociaban el socorro a las economías locales a concesiones privadas, siendo aprovechadas por el capital de los nuevos "colonizadores". Esa fue una derivación de una concepción económica e ideológica de grandes economías, como Inglaterra de Margaret Thatcher; recuerda Helm (2019) que los principales argumentos a la privatización eran la mejor capacidad administrativa y de captación de inversiones. Fue el momento de revisión de los criterios de universalidad, con el regreso de abismos sociales y regionales cuanto al acceso.

Orta (2018) se refiere como el cierre de ciclo el nacimiento del siglo XXI con un proceso de reestatización de las prestadoras de servicios. Sin embargo, no más por la "lógica racional administrativa", pero si bajo un "concepto de corporatización", una prestación por el Estado con características y principios empresariales. Casa (2013) confirma que a mediado de la década del año dos mil, un gran número de servicios ha sido reestatizado, y que, mientras tanto, fue tiempo de normas atractivas a la inversión extranjera y privatización, con la liberalización, la descentralización, participación ciudadana, y la creación de "mercados del agua".

Ocurre que el ciclo histórico se presenta más corto. En la realidad brasileña, en el primer cuarto de siglo, el modelo privatista vuelve con fuerza, interesado en los sistemas superavitarios (una selección permitida por la titularidad municipal de lastre constitucional), e imponiendo el riesgo de un sistema hibrido en que el Estado tenga que subsidiar sistemas deficitarios sin base presupuestaria relacionada a los servicios.

Eso se muestra más evidente, aún, con la recién aprobación por el Senado de la República del Proyecto de Ley No. 4162/2019 (Ley No. 14026/2020), que impide el convenio de los municipios con el Estado, y la decurrente contratación de las respetivas compañías estatales de saneamiento sin licitación, cuando haya el interese de una empresa privada que atienda a una llamada pública obligatoria en el caso de nuevos contractos (a 
partir del año 2022). Una forzada licitación que atenderá solamente a los municipios con sistemas superavitarios.

\section{EL MODELO DE GESTIÓN DE SANEAMIENTO EN BRASIL}

En el ordenamiento jurídico brasileño, por saneamiento se lo tienen los servicios abastecimiento de agua potable, alcantarillado, drenaje pluvial, limpieza urbana y residuos sólidos (art. $3^{\circ}$, de la Ley Federal No. 11445 - Congreso Nacional, 2007).

Tomaremos por saneamiento los servicios de agua y alcantarillado, que son las concesiones comunes y casadas en Brasil (en regla, el drenaje no es concedido, y los servicios de coleta e destinación de residuos sólidos son concedidos separadamente).

Desde el año 1967, la Constitución brasileña atribuye titularidad a los municipios todos los servicios de intereses locales, tal como la actual vigente, del año 1988, en su artículo 30, incisos I y V. Aunque detenga titularidad, la competencia para el establecimiento de directrices y programas, por una necesidad de solución sinérgica, es común con la Unión (Gobierno Federal) y los Estados de la federación (Gobiernos Regionales) - a ejemplo de los artículos 21, XX, y 23, IX, de la Constitución de 1988.

Actualmente (desde el año 2013), mientras tanto, la titularidad exclusiva municipal fue superada en las regiones metropolitanas y aglomeraciones urbanas después del juzgamiento de la Acción Declaratoria de Inconstitucionalidad No. 1842 por el Supremo Tribunal Federal (la Corte Constitucional). Aunque sin grandes efectos prácticos hasta el momento, es superada la cuestión que el interés local sucumbe al regional, no obstante aún no tengamos reglamentación.

Sobre el modelo de gestión, fue solamente a partir del año 1950, con el impacto da la industrialización y la consecuente concentración urbana, a través de una explosión demográfica asociada, y el éxodo rural, que la necesidad de una mayor profesionalización y organización del sector, con soluciones hasta entonces descentralizadas, aisladas e ineficientes, fue presentada a los gestores públicos (Bonemer, 2019), favorecidas incluso por la formación de economías de densidad (Rossoni et al., 2020).

Pero sería solamente en la década de 70, específicamente en el año 1971, que el Gobierno Federal sistematizaría todas las informaciones y necesidades del sector, con la creación del Plano Nacional de Saneamiento Básico, el PLANASA, que permitiría notables conquistas hasta su derrocada en la apertura de la década de 90, más precisamente con la extinción del Banco Nacional de Habitación (BNH), fuente mayor de recursos. En síntesis, en el PLANASA eran atribuciones del Gobierno Federal, Estados y Municipios:

a) Unión (Gobierno Federal): responsable por el establecimiento de directrices generales y el financiamiento de obras estructurales, por medio de recursos nacionales y fuentes internacionales;

b) Estados de la federación: para una racionalización empresarial de los recursos humanos y materiales (Souza, 2016), crearían estructuras para intermediar el recibimiento de los recursos y establecer un modelo profesional y solidario para disminuir los desequilibrios regionales. La solución uniforme fue la creación, por cada Estado, de una Compañía Estadual de Saneamiento Básico (CESB), en forma 
de economía mista (aunque, en la práctica, fuesen mayoritariamente estatales), que por fuerza legal, han asumido el formato de sociedad anónima.

c) Municipios: considerados titulares de los servicios de saneamiento, podrían optar por la adhesión al modelo del Plan Nacional de Saneamiento Básico, o seguir administrando aisladamente, por estructuras propias de la administración directa, o por empresas municipales, o mismo por concesión privada. Con la adhesión al modelo, ocurría la concesión a la compañía del Estado, permitiendo acceso a un capital humano más amplio y a inversiones federales y estaduales direccionadas exclusivamente en al ámbito del programa. La concesión se daba por medio de "convenios" (pero verdaderamente "convenios-contractos") sin necesidad de licitación, y con plazo medio de 30 años. En Brasil, más de 4 mil municipios, de los 5570 (datos de 2015 del Sistema Nacional de Informaciones sobre Saneamiento SNIS) siguen en asociación en ese modelo de gestión regional.

Con la superación de un modelo de gestión hasta entonces sin una capacidad de autosuficiencia, o sin métricas empresariales y con un gigantesco retraso, el Plan Nacional de Saneamiento Básico ha permitido una evolución significativa y que ha resultado en las conquistas estructurales que aun hodiernamente sustentan la gran mayoría de los sistemas, especialmente en las pequeñas y medias ciudades. Por el modelo de gestión estadual, la práctica del subsidio cruzado ha permitido - de una forma de gestión solidaria - que el superávit de grandes sistemas ayudase la construcción y manutención de los pequeños, deficitarios.

Sin embargo, el agotamiento de fuentes de recursos federales - especialmente con la extinción del BNH en el año 1986 - ha impuesto el fallecimiento del PLANASA, dejando los estados y sus respetivas CESBs muchos años sin una efectiva sistematización de inversiones públicas federales en el sector, notado un tímido regreso con el Plano de Aceleración del Crecimiento (PAC) a partir del año 2008.

No se puede olvidar que el Plan Nacional de Saneamiento Básico nace en inicio de los 70, y las concesiones pasan a encerrar en los años 2000, básicamente, ya con los efectos de la falta de inversiones federales. Como veremos más adelante, es momento de un fenómeno de salida de muchos municipios del modelo estadual.

Como contrapartida preparatoria al nuevo momento, el Gobierno Federal establece un nuevo marco legal al sector, con la sanción de la Ley Federal No. 11445/2007 que impone, en su artículo $2^{\circ}$, principios como: universalización del acceso; integralidad; adecuación a la salud pública y medio ambiente; respeto a las peculiaridades locales y regionales; articulación con otras políticas públicas (como desarrollo urbano y regional, habitación, combate a la pobreza, protección ambiental, recursos hídricos, salud y calidad de vida); eficiencia y sustentabilidad económica; adopción de tecnologías adecuadas; transparencia de acciones; controle social; seguridad; calidad; regularidad; y adopción de medidas de fomento al consumo racional.

Señala el legislador, como presentado, con insistencia en la secuencia del texto legal (a ejemplo del art. 11, IV), que los servicios no pueden asumir una postura asistencialista o estar a disposición de arreglos políticos, pero si deben asumir, así como en los 
contractos, una evidente autosuficiencia o sustentabilidad de acuerdo con las disposiciones tarifarias. Es un servicio público esencial, sino con una cara económica, propia de mercado, y que ahora cuenta con la figura del agente de regulación por imperativo de este nuevo marco legal.

Con la extinción de las concesiones realizadas en los 70, hubo un ambiente intensamente litigioso entre municipios y empresas estaduales, con discusiones que siguen actuales sobre aspectos indemnizatorios, relacionados a los activos no amortizados o depreciados. Muchos han optado por un camino aislado, creando empresas municipales o sectores especializados, o haciendo la concesión al sector privado. La gran mayoría, sin embargo, sigue haciendo hincapié en el modelo de gestión estadual, en sinergia entre gobierno municipal y estadual, apostando en el gaño de escala y en la posibilidad de accesos a fuentes de inversiones propias del subsidio cruzado o de la capacidad de endeudamiento de las grandes compañías estaduales.

Aunque la legislación tenga facilitado la sinergia entre Estados y municipios (por conjugación entre el art. 241 de la Constitución, el art. $8^{\circ}$ de la Ley n. 11445/2007, y el art. 24, XXVI, de la ley de licitaciones - Ley n. 8666/93), permitiendo que las Compañías estaduales sean contractadas sin licitación (desde que precedido de convenio de cooperación para la gestión asociada entre Estado y municipio), hay un crecente acoso para escapar de eso modelo solidario, concluyendo algunos gestores municipales que el superávit de su sistema debe alimentar en la totalidad el propio municipio, lo que puede se presentar como una ilusión por la desconsideración de economía de escala, y por lo facto de que en las privatizaciones el lucro no es más socializado, y si incorporado al patrimonio del emprendedor. El escenario inminente es aún más prohibitivo: el Proyecto de Ley Federal No. 4162/2019, recién aprobado por el Senado de la República, trata de impedir la contratación de las estaduales sin licitación cuando presentes interesados privados en las llamadas públicas previas y obligatorias (lo que conducirá una concentración mayor de los sistemas superavitarios con los privados).

Por lo que deprende, entonces, mismo con el fin del Plan Nacional de Saneamiento Básico, los servicios de saneamiento siguen administrados de tres maneras esenciales: $1^{2}$ ) por medio de la cooperación entre municipio y Estado por su respetiva compañía estadual (CESB), que es el modelo mayoritario; $2^{a}$ ) directamente por los propios municipios, por empresa municipal o por sector especializado de la administración directa (más común en pequeñas ciudades); $3^{a}$ ) por concesión al sector privado, por licitación en la modalidad concurrencia pública (aunque muchos gestores han optado por la simples tercerización de los servicios, a perjuicio de los servicios y del interese público).

\section{EL SUBSIDIO CRUZADO ENTRE MUNICIPIOS}

Bonifaz y Montoya (2013) dan a conocer que el esquema de subsidios cruzados en el sector de saneamiento se aplica en la mayor parte de la región de América Latina, que consiste teóricamente en cobrar tarifas por debajo de los costos a un grupo de usuarios y cobrar tarifas por encima de los costos a otro grupo de usuarios, para un impacto distributivo hacia los más pobres. Hay que se comprender que en el campo de un derecho humano, que representa los servicios de saneamiento, los usuarios deben ser considerados en cuanto ciudadanos, y no cliente de servicios (Rossoni et al., 2020). 
La práctica de subsidios atiende a una variada gama de problemas, relacionada a los subsidios entre productos (servicios de agua y alcantarillado cobrados conjuntos), entre consumidores (tarifas regulares, sociales, comerciales etc.), y entre municipios o ciudades (Cruz y Ramos, 2016). Acá tratamos de esa última herramienta de gestión, y por eso no son todos los paradigmas relacionados a la aplicación de subsidios que se muestran válidos, pues dependiente directamente de la forma que cada país trata la titularidad de los servicios y da existencia de una administración regional, o mismo nacional, de los servicios.

Cruz y Ramos (2016) permiten comprender el modelo de subsidio cruzado que coincide con el modelo nacional de gestión de saneamiento creado en el año 1971 con el Plan Nacional de Saneamiento Básico - PLANASA, adherido por los Estados (gobiernos regionales) y los municipios. Con un estudio basado en el método cualitativo, pero con datos económicos de todas las compañías estatales del país, presentan la realidad de los subsidios cruzados aplicados en el sector.

Consideran la existencia, como adelantado, de subsidios cruzados entre productos (diferencia de costos y precios entre servicios de agua y alcantarillado), entre consumidores (residenciales y comerciales, aquellos que consumen poco y consumen más), y, lo que es más relevante al tema, entre municipios (sistemas superavitarios y deficitarios que se equilibran). Evidencian se tratar de un modelo que establece precios artificiales sin alineamiento con el valor de mercado, sin un consenso sobre su utilización, aunque mayoritariamente se apunte como herramienta fundamental a la universalización o expansión de los servicios, en respeto a las disparidades regionales.

Es por medio del subsidio cruzado entre regiones que es posible tornar proyectos que aisladamente serian inviables en virtud del costo de operación y manutención de los sistemas locales (Cruz y Ramos, 2016). Sostienen los autores que una evidencia de esa importancia en la realidad brasileña, reside en la información que diez años después del PLANASA, el índice de abastecimiento de agua ha saltado de 45\% para 86,3\%. Presentase, pues, como un modelo más afecto a la justicia social, una vez que los lucros de los grandes sistemas superavitarios pasa de las manos de los empresarios o inversores a del propio servicio, destinado al socorro de los deficitarios.

El modelo es sostenible por la práctica de una tarifa uniforme, incluso cuando los diversos municipios asociados a una compañía estadual sean heterogéneos en los costos para el suministro de agua y depuración de alcantarillado, visto que para el proceso de captación, depuración, aducción, almacenamiento y distribución de un sistema, no son constantes entre regiones geográficas, a consideración de factores como topología, geología, tipos de fuentes hídricas, calidad de agua, escala, densidad, pierdas del sistema, entre otros que reflejan en el sistema tarifario.

En este escenario, consideran que el sistema vive con inseguridad, pues una vez que la titularidad de los servicios es municipal (a excepción de regiones metropolitanas y aglomerados urbanos, por decisión de la Corte Constitucional, el Supremo Tribunal Federal), los municipios superavitarios pasan a desconsiderar su participación en la misión de justicia social del Estado, optando pela manutención del superávit en sus fronteras. De ahí que encierran pela necesidad de una definición de titularidad al Estado. 
Borja (2014) revela que solamente en el año 2007 ha encerrado un largo período de indefinición del marco legal (con la Ley Federal No. 11445/2007), instaurando una nueva fase de gestión de los servicios de saneamiento en el país, con el planeamiento asumiendo una posición central en la conducción y orientación de la acción pública, considerando que hasta entonces, las inversiones no dialogaban bien con el perfil de los déficits de los servicios. Incluso revela tensiones y contradicciones inherentes a un contexto políticoideológico que mantiene vínculos profundos con la estructura de poder y las relaciones sociales propias de un modelo de producción capitalista. Su trabajo enfrenta las fuentes de financiamiento del Gobierno Federal entre los años 2003 y 2010.

En verdad, mismo con el nuevo marco legislativo del sector (especialmente la Ley Federal No. 11445/2007 - que ahora va a ser actualizado con la aprobación del Proyecto de Ley No. 4162/2019) imponiendo la sustentabilidad económica de los sistemas, y la implementación de tarifas regionalizadas, o que para algunos representa el rompimiento del subsidio cruzado (Guimarães et al., 2014), el modelo de gestión regionalizada o estadual seguía los principios de la legislación de origen del PLANASA.

En el año de 1978, por la Ley Federal No. 6528/1978 (revocada por la Ley Federal No. 11445/2007), se le señalaba que la fijación tarifaria deberá considerar la viabilidad del equilibrio económico-financiero de las compañías estaduales de saneamiento y a la preservación de los aspectos sociales de los servicios, de forma a asegurar el adecuado atendimiento de los usuarios de menor consumo, con base en tarifa mínima (art. $4^{\circ}$ ).

O sea, la fijación tarifaria consideraba el equilibrio de la administradora regional o estadual, y no del sistema local aislado. Por el artículo $2^{\circ}$, había previsión que era el Estado, por su compañía de saneamiento, quien debería realizar estudios para la fijación de las tarifas, según las bases del Ministerio del Interior, conforme el reglamentado en el Decreto Federal No. 82587/1978, que en su artículo 12 reforzaba que la estructura tarifaria debería posibilitar el equilibrio de las compañías, y no de una concesión especifica.

La ley del saneamiento (Ley Federal No. 11445/2007), mientras tanto, pasa a considerar que la política de subsidios no se encuentra en las condiciones de sustentabilidad y equilibrio económico-financiero de la prestación de servicios, que deben ser consideradas en el ámbito de cada contrato de programa, o de cada concesión (art. 39, $\int 2^{\circ}$, c, del Decreto Federal No. 7217/2010).

La política de subsidios, por su vez, es alzada al plan de competencia de la figura de la entidad de regulación (municipal, estadual o intermunicipal), de acuerdo con los

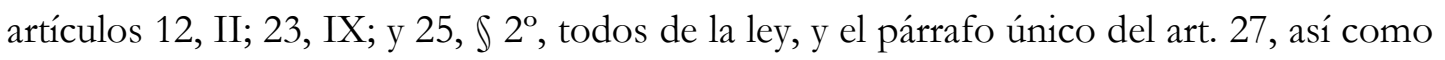
de los artículos art. 30, II, j, y 44, \2 $2^{\circ}$, II, todos del decreto No. 7217/2010, regulatorio de la ley. La regulación, importa registrar, es directamente afectada por el recién aprobado Proyecto de Ley No. 4162/2019, pasando a atender una reglamentación nacional, por la Agencia Nacional de Aguas.

Aunque la ley de 2007 trate da posibilidad de subsidios entre localidades (art. 29, § $2^{\circ}$, y art. 31, III), y reconozca conceptualmente que subsidio es un instrumento de política social para garantizar la universalización del acceso al saneamiento básico, especialmente para población y "localidades" de bajos ingresos (art. $3^{\circ}$, VII), el sentido regulatorio es de 
que el superávit de un sistema debe servir a la expansión del mismo, así como para la minoración de las tarifas cuando ocurra la universalidad, lo que coincide con la conclusión de Yepes (2003) de que las empresas deben generar un flujo de caja positivo que les permita financiar los costos de expansión de los servicios, sea cual el modelo de subsidio aplicado.

\section{EL IMPACTO DEL MODELO EN EL ESTADO DE SANTA CATARINA}

Santa Catarina es un Estado de la federación con un índice de desarrollo humano mejor que la media nacional (IDH - 0,774), y mejor que muchos países con un alto IDH, tal como Perú con su índice de 0,725. Tiene una población estimada de poco más de 7 millones de habitantes, dividida en 295 municipios, y representa el cuarto mayor Producto Interno Bruto (PIB) de Brasil.

En tema de saneamiento, en lo que importa los servicios de agua, Santa Catarina ubicase entre los 18 Estados, de las 27 unidades de la federación (26 Estados y un Distrito Federal), con más de $90 \%$ de cobertura de atendimiento urbano, de acuerdo con el Sistema Nacional de Informaciones sobre Saneamiento - SNIS.

El índice de desarrollo humano positivo es consecuencia, también, de esa condición, mientras que esa condición de suficiencia es respuesta de la capacidad de inversiones decurrente de una economía próspera, dependiente directa de una capacidad de recursos hídricos. Es facto que hay una correlación directa entre desarrollo económico y capacidad o suficiencia de los servicios de abastecimiento de agua potable. Los Estados del sur y sudeste presentan evidente distinción positiva con los de la región norte.

Mientras tanto, no hay una correspondencia relacionada con los servicios de coleta y depuración de alcantarillado o aguas residuales. Mismo con un Producto Interno Bruto ascendente e imponente, en relación con las demás unidades de la federación, Santa Catarina no ha contestado de igual manera en comparación con los servicios de agua. Brasil ha evidenciado un retraso muy significativo en ese tema, y Santa Catarina aún más en razón de su distinción - positiva - económica y social.

De acuerdo con el Sistema Nacional de Informaciones sobre Saneamiento (2018), el índice medio de atendimiento urbano en saneamiento básico apuntaba solamente tres Estados superiores a 70\% de cobertura (São Paulo, Paraná y Minas Gerais). Santa Catarina encontrándose en la incómoda tercera escala de Estados, entre 20\% a 40\%.

En el año 2010, Santa Catarina estaba posicionada en la incómoda $23^{a}$ posición (entre las 27 unidades de la federación) en el ranking de cobertura de efluentes. En la última década, después de aportes federales (PAC), internacionales (instituciones de fomento de Japón - JICA, y Francia - AFD), y de una mayor profesionalización del sector, en el año 2018 ya caminaba para la $13^{a}$ posición. Sin embargo, seguimos tratando de un Estado que tiene uno de los mejores IDH, y uno de las mayores economías (la cuarta) del país. Una evidente contradicción.

La gestión de los servicios de saneamiento en el Estado de Santa Catarina sigue el modelo nacional, especialmente a partir de la institución del Plan Nacional de Saneamiento Básico (PLANASA). Fue en ese ambiente, que en el año 1971 fue creada por ley estadual (No. 4547) la Compañía Catarinense de Aguas y Saneamiento (CASAN), 
una sociedad de economía mista (aunque con más de 99\% de capital público), con el objetivo de coordinar el planeamiento y ejecutar los servicios de saneamiento (agua y alcantarillado), con formalización de convenios con los municipios del Estado.

Con su creación, para socorro al modelo y recursos del Plan Nacional de Saneamiento Básico, la gran mayoría de los 295 municipios fueron adherentes, firmando convenios de treinta años. Sin embargo, algunos pocos han optado por un camino propio, manteniendo los servicios bajo administración puramente municipal, como se puede citar: Blumenau, São Ludgero, Orleans, Jaraguá do Sul. En aquel momento, la privatización del sector no se constituya en una realidad próxima.

Como la participación federal en el PLANASA no ha sido como prometida (aporte de suficientes recursos), y especialmente con su ruina en la apertura de los años 90 , hubo perjuicio en la realización de inversiones en el sector, con destaque para el servicio de coleta, transporte, depuración y disposición final de alcantarillado o aguas residuales. Es que el Estado de Santa Catarina es formado mayoritariamente por municipios pequeños (39 con menos de 5 mil habitantes), y con presupuestos o facturación de tarifas insuficiente para hacer frente a las necesidades.

En el ambiente de gestión estadual, con el subsidio cruzado, la Compañía estadual tuvo que proporcionar ayuda a la mayoría de los municipios deficitarios, con los pocos superavitarios; un modelo solidario de corrección de distorsiones regionales, pero que necesitaba del apoyo gubernamental para inversiones en obras de estructuración.

Con el vencimiento de las concesiones a partir del descortinar del siglo XXI, un conjunto de factores ha impactado en la manutención del modelo en muchos e importantes municipios como: 1) la insatisfacción con el modelo de subsidio cruzado y con los resultados de los servicios obtenidos al largo de la concesión; 2) proyectos políticos distintos entre alcaldes y gobernadores; 3) presión del sector privado, una realidad local diversa de aquella de los años 70 .

De ese conjunto, municipios importantes como Joinville, Palhoça, Balneário Camboriú, Itajaí, Timbó, Penha, Itapema, Sombrio, Lages entre otros más chicos han optado por caminos distintos. Con ese fenómeno, actualmente 195, de un total de 295 municipios catarinenses, han permanecido bajo gestión asociada con el Estado.

Sin embargo, aunque los 195 municipios representen 66\% de los del Estado, representan solamente $45 \%$ de la población, un indicativo de que las iniciativas de administración aisladas o privatizadas alcanzan los mayores y los más atractivos económicamente, impactando en la pérdida de fuerza del subsidio cruzado (además del impacto en el activo de la compañía del Estado - más de R \$ 500 millones).

Eses números no consideran, aún, los municipios que han combatido los contratos en su curso, con o sin éxito de rescisión. La mayoría de aquellos que han cambiado de modelo han elegido una administración por estructuras propias, y pocos han efectivamente hecho una concesión clásica al sector privado.

Muchos, con la abrupta salida de la empresa estadual, han hecho - con muy cuestionable legalidad - contrataciones por emergencia, sin licitación, que se han prolongado por años, con resultado negativos a la expansión de los servicios, o hecho 
solamente la tercerización, que incluso no atiende a los nuevos requisitos de profesionalización del sector.

\section{RESULTADOS Y DISCUSIÓN}

Es verdad que algunos municipios que no han adherido al modelo del Plan Nacional de Saneamiento Básico en la década de setenta han conquistados muy buenos índices de cobertura en agua y alcantarillado (São Ludgero y Jaraguá do Sul), en función de fuentes federales destinadas a los municipios (especialmente por la Fundación Nacional de Salud - FUNASA), y también por exitosas gestiones; sin embargo, lo mismo no puede ser observado en los municipios que han abandonado el modelo de gestión asociada después de los término de las antiguas concesiones, a partir de una comparación del volumen de inversiones y de las obras estructurantes observadas en cada modelo.

Mismo con la presión en contra al modelo de administración regional, sigue siendo modelo único de momento a permitir la corrección de distorsiones regionales y de justicia social en lo que toca al alcance a esos derechos humanos; mientras tanto, es cierto que la modicidad tarifaria necesaria, la perdida de sistemas superavitarios y el subsidio cruzado aplicado entre los servicios (agua y alcantarillado) y los consumidores (tarifas sociales, residenciales, comerciales, industriales y públicas) no han admitido seguridad a permitir la universalidad de los servicios sin el aporte de recursos externos, especialmente públicos (Estado y Gobierno Federal), escasos en la actualidad.

En tanto los autores Paludo y Borba (2013) han producido un estudio comparativo entre dos municipios del Estado de Santa Catarina: uno adherente al modelo de gestión asociada con el Estado (CASAN), que es Indaial; otro que ha abandonado el modelo, adoptando la privatización, que es el caso de Itapema. Fueron considerados diversos aspectos, desde la localización geográfica, el tamaño de las ciudades, aspectos históricos, niveles socioeconómicos, políticas ambientales y contextos políticos. La investigación ha observado que las tarifas se presentaron más altas en el modelo privatizado y que en el aspecto financiero, en el modelo público toda la recaudación se invierte en el sistema, cuando el lucro es apropiado en el sector privado; además, destaca que en la gestión asociada municipio-estado hay una mayor consideración con el aspecto social y con las poblaciones más carentes. Concluyen que:

Diante da pesquisa empírica aqui apresentada, as principais diferenças não estão nos resultados econômicos (custo e tarifa) ou técnicos (metas de acesso), mas especialmente em relação aos aspectos ambientais e de inclusão e participação social, os quais são levados em consideração pelo modelo de gestão compartilhada como objetivos estratégicos a serem alcançados (Paludo y Borba, 2013, p. 72).

Otra comparación puede ser realizada entre dos grandes municipios: Florianópolis y Palhoça. El primero, ha permanecido en el modelo de gestión pública asociada, en cuanto el segundo ha optado - en el año 2008 - por la privatización, a través de la tercerización.

En 2008, Florianópolis contaba con una cobertura de coleta y depuración de alcantarillado en la orden de 45\%; en el año 2020, ha ampliado para casi 70\%. Palhoça, establecida en la misma región metropolitana, y entre los diez mayores municipios del 
Estado, ni siquiera tiene una red pública central de saneamiento básico con coleta y depuración, mismo con una de las mayores recaudaciones tarifarias.

\section{CONCLUSIÓN}

El reconocimiento internacional del saneamiento en cuanto derecho humano, es considerado como servicio público y esencial, aunque delegable al privado. Sin embargo, hay acierto en la conclusión de Colin et al. (2016) de que ese reconocimiento no ha sido una "mágica" a permitir que los países atribuyesen más consideración o inversiones sobre los servicios relacionados.

El modelo brasileño, contrariando otras realidades del continente que impiden la privatización de los servicios de saneamiento (Bolivia, Ecuador y Uruguay, $v . g$.), admite y ahora incentiva - o mismo impone - bajo una plataforma abiertamente neoliberal, con la aprobación de la Ley Federal No. 14026/2020. Mientras tanto, el modelo reinante sigue siendo el de la gestión asociada, por convenios entre Estados de la federación y municipios, que resultan en la contratación de las compañías estaduales.

Hay regulación, participación social y posibilidad de mayor justicia social con el empleo del subsidio cruzado, que corrige distorsiones regionales. Hay una óptica de prevalencia social sobre el lucro, aunque el equilibrio económico sea una prescripción legal.

El modelo de gestión como una alternativa privada exige extrema precaución, una vez que hablamos de un derecho humano, y "en la medida en que una empresa adquiere los derechos sobre el agua, dígase, en una ciudad, las necesidades vitales de la población de esta ciudad pasan a sujetarse a las estrategias de maximización de la empresa", y "el control monopolístico del agua en la ciudad le confiere a la empresa privada poder sobre la vida de la ciudadanía" (Villa Fontecha, 2012, p. 124).

En el caso paradigma, del Estado de Santa Catarina, se percibe la utilización de los marcos legales (como la Ley Federal No. 11445/2007 - Ley del Saneamiento - y la Ley Federal No. 13303/2016 - Ley de las Estatales) para superar sus contradicciones relacionadas con la capacidad de respuesta al pasivo en materia de coleta y depuración de alcantarillado, replicando el modelo nacional de gestión asociada.

Para la conquista de su plenitud, sin embargo, solamente las tarifas no serán suficientes, sin violar el principio de modicidad tarifaria de garantía legal, siendo necesario que los coobligados por imposición constitucional cumplan con su papel, aportando recursos, y una mayor seguridad jurídica para los contratos.

Esa es una constatación también decurrente de la práctica de los diversos tipos de subsidios practicados en el continente (entre usuarios, productos y localidades o ciudades). Bonifaz y Montoya (2013) advierten que, en la práctica, la alternativa de los subsidios cruzados no ha cumplido cabalmente con los objetivos planteados.

Es posible advertir que el fenómeno de la privatización es una realidad cada vez más presente en el escenario brasileño y especialmente en el Estado paradigma, contrariando la regla general de reestatización planteada por Orta (2018) para el inicio del siglo XXI. De ahí, necesario que la cúpula administrativa de la CESB y del Estado sea 
consciente de las verdaderas razones de este fenómeno, exigiendo soluciones que pasan por la reestructuración delante los riesgos relacionados a la supresión legislativa del modelo de gestión por subsidio cruzado o solidario. En lo contrario, surge recomendaciones - o necesidad - de subsidios directos (Mejía y Valverde, 2017), oriundos del presupuesto estatal.

La tarea de universalización del saneamiento básico en Brasil involucra un amplio esfuerzo político-ideológico de los diversos seguimientos de la sociedad civil organizada, en el sentido de llevar las acciones de saneamiento básico para el campo del derecho social, integrante de una política pública sustentada en una profunda acción de Estado. Ese aspecto se presente muy relevante para las consideraciones objetivas y subjetivas entre la participación pública y privada en el mercado.

\section{REFERENCIAS BIBLIOGRÁFICAS}

Aith, F. (2010). Saúde e saneamento no Brasil: aspectos conceituais e regulatórios e os desafios para a adoção de políticas públicas intersetoriais no país. En Mota, C. (org.). Saneamento básico no Brasil: aspectos jurídicos da lei federal n 11.445/07. Rio de Janeiro: Quartier Latin.

Assembléia Nacional Constituínte. (1988, 5 de octubre). Constituição da República. Diário Oficial da União, Seção 1, p. 1, Brasil.

Bel, G. (2020). Public private water delivery, remunicipalization and water tariffs. Utilities Policy, 62 (2), Estados Unidos. DOI: https://doi.org/10.1016/j.jup.2019.100982

Bonemer, B. (2019). Do PLANASA à gestão associada: a modelagem jurídica e contábil aplicada às relações entre as companhias estaduais de saneamento básico (CESB's), os Estados-Federados e os Municípios convenentes. En Marcos, H. V. (Org.). Saneamento e estatais: temas jurídicos contemporâneos. Florianópolis: EMais Editora.

Bonifaz Fernández, J. L, Montoya Villavicencio, J. J. (2013). Propuesta para mejorar la progresividad del subsidio cruzado al agua potable en SEDAPAL. http:/ / repositorio.up.edu.pe/handle/11354/432

Borja, P. C. (2014). Política pública de saneamento básico: uma análise da recente experiência brasileira. Saúde e Sociedade, 23 (2), pp. 432-447. DOI: https://doi.org/10.1590/S0104-12902014000200007.

Borja-Vega, C., Kloeve, E. (2018). Why a human rights-based approach to water and sanitation is essential for the poor. https://blogs.worldbank.org/water/whyhuman-rights-based-approach-water-and-sanitation-essential-poor

Brown, C., Neves-Silva, P., \& Heller, L. (2016). The human right to water and sanitation: a new perspective for public policies. Ciência \& Saúde Coletiva, 21 (3), pp. 661-670. https://doi.org/10.1590/1413-81232015213.20142015

Casa, M. (2013). Capacidades estatales para una gestión participative del agua: experiencias del caso uruguayo. Revista Uruguaya de Ciencia Política, 22 (1), pp. 103-128. http://www.scielo.edu.uy/scielo.php?script=sci_arttext\&pid=S1688-499X201300 0100005\&lng=es\&tlng $=$ es 
Ceppi, N. (2014). La política exterior de Bolivia en tiempos de Evo Morales Ayma. Si Somos Americanos, 14 (1), pp. 125-151. DOI: https://dx.doi.org/10.4067/S071909482014000100007

Congresso Nacional. (1978, 12 de junio). Lei n. 6.528, de 11 de maio de 1978. Diário Oficial da União, Seção 1, p. 6951. Brasil.

Congresso Nacional. (2007, 8 de enero). Lei n. 11.445, de 05 de janeiro de 2007. Diário Oficial da União, Seção 1, p. 3. Brasil.

Congresso Nacional. (2016, $1^{\circ}$ de julio). Lei n. 13.303, de 30 de junho de 2016. Diário Oficial da União, Seção 1, p. 12. Brasil.

Congresso Nacional. (2019). Projeto de Lei n. 4162/2019. https://www.camara.leg.br/proposicoesWeb/fichadetramitacao?idProposicao $=2$ 207613

Cruz, K. A., Ramos, F. S. (2016). Evidências de subsídio cruzado no setor de saneamento básico nacional e suas consequências. Nova Economia, 26 (2), pp. 623-651). DOI: https://doi.org/10.1590/0103-6351/2544

Dia Mundial do Meio Ambiente: plano de investimentos da CASAN avança esgotamento sanitário em SC (2019, 4 de junio). Casan. https://www.casan.com.br/noticia/index/url/diamundial-do-meio-ambiente-plano-de-investimentos-da-casan-avanca-esgotamen to-sanitario-em-sc\#0

Guimarães, E. F., Coutinho, S. M. V., Malheiros, T. F., \& Philippi Jr., A. (2014). ¿Os indicadores do saneamento medem a universalização em áreas de vulnerabilidade social? Engenharia Sanitaria e Ambiental, 19 (1), pp. 53-60. DOI: https://doi.org/10.1590/S1413-41522014000100006

Helm, D. (2019). Thirty years after water privatization—is the English model the envy of the world? Oxford Review of Economic Policy, 36 (1), pp. 69-85. DOI: https://doi.org/10.1093/oxrep/grz031

Graf, A. (2008). A tutela dos estados sobre as águas. En Freitas, V. P. (Coord.). Águas: aspectos jurídicos e ambientais ( $3^{\mathrm{a}}$ edición). Curitiba: Juruá.

Mejía, A., Uzcátegui, G., Valverde, O. (2017). Agua y saneamiento en el Estado Plurinacional de Bolivia. http://scioteca.caf.com/handle/123456789/1081.

Ministério do Desenvolvimento Regional (2018). Sistema Nacional de Informações sobre Saneamento: $24^{\circ}$ Diagnóstico dos Serviços de Água e Esgotos - 2018. http://www.snis.gov.br/downloads/diagnosticos/ae/2018/Diagnostico_AE2018 .pdf

Orta, M. (2018). La gobernabilidad de los servicios de agua y saneamiento y los conflictos por el agua en América Latina. Journal De Ciencias Sociales, 6 (11), pp. 148-170. DOI: https://doi.org/10.18682/jcs.v0i11.793

Poder Executivo (1978, 7 de noviembre). Decreto n. 82.587, de 6 de novembro de 1978. Diário Oficial da União, Seção 1, p. 17830, Brasil. 
Poder Executivo (2010, 22 de junio). Decreto n. 7.217, de 21 de junho de 2010. Diário Oficial da União, Seção 1, Edição Extra, p. 1, Brasil.

Rossoni, H. A. V., Faria, M. T. S., Silva, A. C., \& Heller, L. (2020). Aspectos socioeconômicos e de desenvolvimento humano municipal determinantes na ausência de prestadores de serviços de esgotamento sanitário no Brasil. Engenharia Sanitária e Ambiental, 25 (2), pp. 393-402. DOI:_https://doi.org/10.1590/s141341522020183887

Sousa, A. C. A., Costa, N. R. (2016). Política de saneamento básico no Brasil: discussão de uma tragédia. História, Ciência, Saúde - Manguinhos, Rio de Janeiro, 23 (3), pp. 615634. DOI: http://dx.doi.org/10.1590/S0104-59702016000300002

Souza, G. S., Faria, R. C., \& Moreira, T. B. S. (2008). Efficiency of Brazilian public and private water utilities. Estudos Econômicos, 38 (4), pp. 905-917. DOI: https://doi.org/10.1590/S0101-41612008000400008

Supremo Tribunal Federal (2013). Ação Direta de Inconstitucionalidade n 1842/RJ. Diário da Justiça Eletrônico n. 47, 11/03/2013, Brasil.

Teixeira, J. C., Oliveira, G. S., Viali, A. M., \& Muniz, S. S. (2014). Estudo do impacto das deficiências de saneamento básico sobre a saúde pública no Brasil no período de 2001 a 2009. Engenharia Sanitária e Ambiental, 19 (1), pp. 87-96. DOI: https://doi.org/10.1590/S1413-41522014000100010

Villa Fontecha, G. H. (2012). A propósito de la gestión del agua en el mundo contemporâneo. Um enfoque biopolítico. Análisis Político, 25 (74), pp. 109-133. http://www.scielo.org.co/scielo.php?script=sci_arttext\&pid=S0121-4705201200 0100006\&lng=en\&tlng $=$

Yepes, G. M. (2003). Los subsidios cruzados en los servicios de agua potable y saneamiento. Banco Interamericano de Desarrollo. https://publications.iadb.org/ publications/spanish/document/Los_subsidios_cruzados_en_los_servicios_de_a gua_potable_y_saneamiento.pdf

\section{CITAR COMO:}

Victor Marcos, H., \& Santa María, B. C. (2021). Importancia de la gestión regional del saneamiento: el paradigma del Estado de Santa Catarina en el uso del subsidio cruzado. Puriq, 3(1), 175-189. https://doi.org/10.37073/puriq.3.1.122 